Invited paper for Fifth International Congress of Radiation Research, 14-20 July. 1974, Seattle, Washington.

\title{
IONIZATION BY INTERPENETRATION OF ELECTRON SHELLS *
}

Felix T. Smith and A. Salop

Molecular Physics center

Stanford Research Institute

Menlo Park, California 94025

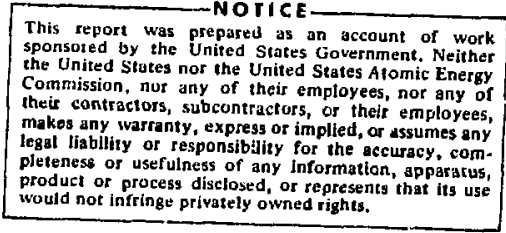

Number of copies submitted: $\quad 3$

Manuscript pages: 15

Number of figures: $\quad 10$

Number of tables: 0

\footnotetext{
*Work supported by the Atomic Energy Commission, Division of Blology and Medicine.
} 
A11 correspondence to be sent to:

Dr. Felix T. Smith Molecular Physics Center Stanford Research Institute 333 Ravenswood Ave. Menlo Park, California 94025 


\section{Abstract}

Smith, Felix T., and Salop, A. Ionization by Internenetration of Electron Shells

Until recently, the prevailing model for ionization by charged particle impact was based on the coulomb interaction of a fast structureless charged particle with the electron cloud of the target. This model is characterized by a very rapid decline in the ionization cross section at low relative velocities (measured by comparison with the electron velocity in the atomic shell being ionized). When higher $z$ particles with IIlled electron shells are used, measured cross sections for ionization are of ten much larger than coulomb cross sections. This is predominantly attributable to the Pauli exclusion interaction between electrons in interpenetrating shells, which causes high promotion and even ionization of one or more of the electrons involved. This ionization process can be predicted from the behavior of quasi-molecular wave functions for the pair of colliding atoms. The same principles generally apply whether the ionization is from deep inner shells of heavy particles or from outer shells, provided the relative velocity is similar. Typical cross sections may be as large as the electron shells involved and may remain high down to threshold energies determined by the appropriate ionization potential, augmented by the effective internuclear coulomb repulsion energy at the distance involved.

Key words: collisional ionization, inner shell excitation, inner shell ionization, heavy particle collisions. 


\section{INTRODUCTION}

At the symposium ${ }^{(1)}$ that preceded this one, eight years ago, Professors Fano and Kistemaker both printed out the potential importance of the innershell ionization and excitation processes arising from the interpenetration of more or less filled electron shells in two coiliding atoms or ions. This subject was then a rather new one to which attention had been drawn by experimental measurements at Leningrad ${ }^{(2)}$ and at the University of connecticut $(3,4)$, and the explanation for these processes in terms of the electron promotion nechanism of molecular orbital theory had just been given by Fano and Lichten (5). In the eight years that have intervened since that time, a great deal of work has been done that has shed light on the details of this process, but it is impressive to look back to the earlier symposium and see how sound were the statements on this topic and the predictions of its importance.

Actually, the interpenetration of electron shells as a source of excitation and ionization is a significant process, even for the outer shells of atoms and molecules--as can be seen in collisions of closed-shell atoms or molecules at energies as low as a few hundred $\mathrm{eV}$. The same process applies at somewhat higher energies (10 or $20 \mathrm{keV}$ and above) for the ionization of inner shells--such as the $K$ shells of $O, N$, and $C$ in biological materials. As we shall see later, these processes may be quantitatively rather significant; perhaps 10 or $20 \%$ of the energy available may be deposited in inner-shell ionization events.

The phenomena under consideration here are confined to a situation where two particles collide, both of which are carrying one or more electrons 

Fastrup $^{(6)}$ and Garcia, Fortner, and Kavenaugh ${ }^{(7)}$ and also by much detailed work reported in the 1972 Proceedings of the International Conference on Inner Shell Ionization Phenomena ${ }^{(8)}$.

\section{COULOMB IONIZATION}

When a charged particle passes through matter, it creates a rapidly changing electromagnetic field. The electrons in the atoms of the medium respond to this time-dependent field and are thereby excited and frequently removed from their immediate environment in an ionization event. This type of ionization is often called coulomb ionization, because it is a simple response to the time-dependent coulomb field. Since a rapidly varying electromagnetic field is involved, coulomb lonization by fast particles bears a close relationship to photoionization, a relationship that has been well exploited in studies of excitation and ionization by fast heavy particles such as protons and alpha particles and even heavier nuclei, especially when they have been substantially stripped of their accompanying electrons. The theory of the coulomb excitation mechanism is very well developed $(7,9,10)$ and will not be discussed in detail here, but it is an important point of departure for comparisons with other theories. According to this model, the ionization cross section peaks at a particle velocity that matches approximately the electron velocity in the atomic shell involved and decreases rapidly with decrease in projectile velocity.

Figure 1 is a plot of experimentally obtained K-shell ionization cross sections versus impact energy for proton and alpha particle impact on various 
heavy target atoms, taken from a recent paper by W. Brandt ${ }^{(9)}$, which 11 lustrates very clearly the general behavior of coulomb ionization.

The experimental results, when plotted in the appropriate reduced units, lie very close to a theoretical universal curve valid in the region where the projectile is slower than the $\mathrm{K}$-shell electron. The universal curve is derived from the predictions of the plane wave born approximation (PWBA), with corrections made for the effects of coulomb deflection of the projectile and the variation of $\mathrm{K}$-shell binding with internuclear distance. The scaling reflects the velocity matching requirements referred to previously and takes into consideration the dependences of the cross sections on projectile and target nuclear charges.

\section{CHARGE TRANSFER IONIZATION}

Charge transfer processes resulting in the production of excited ions have already been discussed in this symposium by Professor Niehaus, and we need not go into details about them here. We need only remember that these processes, when they are surficiently exothermic, may have large cross sections, even down to the lowest velocities of collision. Furthermore, a multiply charged borabarding ion may rob an atom or molecule of an electrun from an inner she11. This event can be followed by an Auger emission of another electron or by an $X$-ray event. The cross sections and energy dependence of these processes are, of course, totally different from the behavior of the coulomb Ionization process. 


\section{SHELL INTERPENETRATION PHENOMENA}

Figure 2 shows how interpenetrating electron shells can cause ionization with a cross section far above that for coulomb ionization. ${ }^{(9,11)}$ It shows the contrast between the bare charged particles (protons, alphas) and simply ionized heavy atoms $(\mathrm{C}, \mathrm{N}, \mathrm{O}, \mathrm{Ne})$. The results for the heavy ions exceed the universal coulomb curve by factors of $10^{4}$ and greater. Clearly, these heavy particles are ionizing predominately by a mechanism entirely different from the coulomb process. The closed inner shells of the collision partners interpenetrate, and $r()^{\circ}$ ces associated with the Pauli exclusion principle are set up during the collision. Some of the electrons are forced into transient, highly excited, quasimolecular configurations during the collision. When the atoms separate, these electrons have a high probability of being left in excited states, often with ample energy to cause ionization in subsequent Auger transitions. This is basically the mechanism for which Fano and Lichten developed their molecular orbital model.

Before we discuss the interpenetration phenomena in more detail, we w111 consider some additional examples of the general disparity between heavy Ion data and the predictions of theories applicable to incident structureless particles. Figures 3 and 4 show some similar cross sections taken from Garcia et al. (7) for excitation of K-, L-, and M-shell electrons during collisions of several heavy lons with various targets. The data are compared with calculation. of point charge coulomb ionization obtained by using binary encounter theory (BEA) 
(roughly comparable with PHBA calculations) and plotted with slightly different reduced units. Again, most of the experimental cross sections are greatly in excess of theoretical values for an incident point charge and reflect the dominance of shell interpenetration ionization.

Figure 5 taken from Ogurtsov's work ${ }^{(12)}$ is a very elegant picture that demonstrates the fundamental difference between the K-shell excitation produced by a heavy ion collision and that due to photon, electron, and proton interactions. Auger electron spectra corresponding to transitions involving an initial $\mathrm{K}$-shell vacancy in neon are shown for excitation produced by $1,5-k e V$ photon, 3.2-keV electron, 300-keV proton, and $200-\mathrm{keV} \mathrm{Ne}^{+}$ion bombardment. The photon and electron spectra are identical, reflecting the fact that the electron excites the $K$ shell via an electromagnetic pulse. In the case of proton excitation, in which the mechanism is similar, the same dominant transitions are noted, but a few other weak lines are observed, which can be attributed to additional excitation in the neon atom produced as a result of the proton interaction. The fourth spectrum for excitation by incident neon ions, however, is dramatically different from the other three. The Auger electron spectrum is considerably smeared out, with a very large increase in the numbers of lower energy electrons emitted, indicating the dominance of L-shell Interpenetration excitation processes. These examples are certainly sufficient to indicate how inportant shell interpenetration effects are with regard to the amount and pattern of energy deposition. They produce large amounts of excitation involving considerable energy transfer which of ten leads to multiple ionization through Auger cascades, electron shake-off, and other reorganizational processes. 
How can these processes be studied in more detail? We have seen several examples already. One can study the X-rays or photons emitted, or one can examine the Auger spectra in experiments that are in a sense "integrated" measurements, giving useful information about the excitation produced over a wide range of scattering angles or impact parameters. However, the experiments that have provided the most insight into inner-shell excitations are differential scattering experiments, particularly those that have been set up to measure the inelastic energy loss of the collision for various scattering angles (or, equivalently, distances of closest approach). These are of ten coincidence experiments in which the charge states of the product ions are also measured. We shall concentrate for the next few paragraphs on the experiments dealing with the collisions of $\mathrm{Ar}^{+}$on $\mathrm{Ar}$, as these very nicely illustrate the shell interpenetration phenomena and are the sort of measurements that originally led to the Fano-Lichten theory.

\section{DIFFERENTIAL INELASTIC WNERGY LOSS MEASUREMENTS}

Figure 6 is a spectrum ${ }^{(13)}$ that shows three well-resolved energy-loss peaks observed in the reaction

$$
\mathrm{Ar}^{+}+\mathrm{Ar} \rightarrow \mathrm{Ar}^{2+}+\mathrm{Ar}^{2+}+3 e
$$

at 25-keV collision energy and $16^{\circ}$ scattering angle. Fano and Lichten first explained this three-peak structure in connection with earlier measurements in their classic 1965 paper. According to their interpretation, $Q_{I}, Q_{I I}$, and $Q_{\text {III }}$ correspond to collisions in which zero, one, and two electrons, respectively, 
are raised out of the inner $L$ shells of either Ar atom, in addition to a variable number of M-shell excitations. Each vacancy tends to be filled by an Auger transition involving two outer electrons. One moves in to fill the vacancy, and the other leaves the atom with an energy slightly smaller than the relevant L-she11 binding energy ( $200 \mathrm{eV})$, which should and does correspond to the energy interva1 between $Q_{I}$ and $Q_{I I}$ and between $Q_{I I}$ and $Q_{I I I} Q_{i}$ peaks at about $180 \mathrm{eV}$, which is insufficient for L-shell ionization but which corresponds to appreciable $M-s h e l l$ excitation. The reason for the appearance of only three peaks will become clear when we discuss the Fano-Lichten model.

By examining these processes as a function of scattering angle at a given collision energy, one can determine the distances of closest approach at which these L-shell excitation processes occur. Figure 7 shows relative probabilities for the inelastic energy-loss events corresponding to $Q_{I}, Q_{I I}$, and $Q_{I I I}$ of Figure 6 as a function of distance of closest approach for 50-keV Ar ${ }^{+}$-Ar col1isions (14) We see that below 0.27 essentially only outer-shell excitation processes are occurring. The onset for L-shell ionization at about $0.27 I_{\text {is }}$ quite sharp, and by $0.20 \AA$ the relative probability of double L-shell excitation is almost $100 \%$

The average inelastic energy loss $\bar{Q}$ vs. the distance of closest approach for $\mathrm{Ar}^{+}-\mathrm{Ar}$ collisions in the range of collision energies from $25 \mathrm{keV}$ to $1.5 \mathrm{MeV}$ is shown in a comprehensive plot in Figure $8^{(15)}$. This figure illustrates clearly the striking increases in excitation that occur when various shells start to interpenetrate, as characterized by various critical values of $R_{\text {of }}$ the distance of closest approach. Thus, the step increases in $\bar{Q}$ observed for the 25-keV data at $R_{0} \approx 0.25 \AA$ occur when the $L$ shells start to interpenetrate, as 


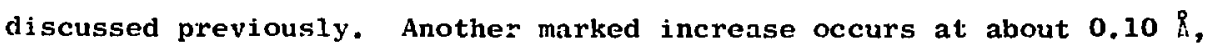
presumably because of the production of additional L vacancies. Finally, an approximately $3-\mathrm{keV}$ increase in $\bar{Q}$ is observed for $R_{0}$ values between 0.02 and $0.01 \AA$. This can be attributed to the interpenetration of the argon $\mathrm{K}$ shells and the consequent production of a $\mathrm{K}$-shell vacancy.

FANO-LICHTEN MODEL

Figure 9 is a reproduction of the diabatic moiecular orbital diagram first presented by Fano and Lichten to illustrate the electron promotion excitation process for the $\mathrm{Ar}^{+}-\mathrm{Ar}$ system. We obviously cannot go into all the details here; a good deal. of 1 iterature is now available on this subject, and the reader is referred, in particular, to Refs 6 and 7 and to the criginal papers of Fano and Lichten. ${ }^{(5,16)}$ With reference to the experimental $\mathrm{Ar}^{+}-\mathrm{Ar}$ data, however, consider collisions in which the energy is high enough so that $L$ shells can interpenetrate, corresponding to internuclear distances somewhat smaller than $0.25 \&$ (or $\sim 0.5$ atomic unit on the Fano-Lichten diagram). It can be seen that the diabatic $4 f_{\sigma}$ molecular oritial, which rises sharply with decrease in internuclear separation in this region, carries two $2 p$ electrons through a series of closely spaced crossings. It is thus likely that transitions will occur and that after the collisions the electrons will find themselves left in highly excited orbitals with one or two I-shell vacancies appearing in the separated argon ions. Ionization through Auger transitions can then follow. Thus, the presence of three excitation peaks and the sharp onset of L-shell excitation at distances of closest approach of about $0.25 \AA$ in the experimental 
data discussed previously are explained by this model. Similarly, the onset of $\mathrm{K}$-shell excitation at about $0.02 \AA$ is explained as due to a transition between the $2 \mathrm{p}_{\sigma}$ and $2 \mathrm{p}_{\pi}$ molecular orbitals, this time through the mechanism of rotational coupling ${ }^{(6)}$, which will be described shortly.

In general, this electron-promotion molecular orbital description of the interaction should have its maximum validity when the orbital energies in the two atoms are not very different and when tine projectile velocity is considerably smaller than the orbital electron velocity in question. Symmetric or almost symmetric collisions form one of the most important cases of the first conditior, but it may apply also in very asymmetric cases, for instance, where the $\mathrm{K}$-shell energies in one atom are close to the L-shell energies in another. The velocity criterion means also that the molecular orbital description may be valid for inner-shell interactions at velocities where it may be totally inapplicable for the outer shells in the same collision. At high velocities in a condensed medium, the bombarding ion soon becomes very highly stripped, and coulomb excitation will probably become the dominant process, although if the ion is heavy, electron capture and loss may also be an important mechanism.

\section{COUPLING MECHANISMS}

We will briefly discuss the various types of energy-level crossings and interactions occurring in these collisions by referring to Figure 10, a schematic representation of the lowest one-electron energy levels of two atoms $A$ and $B$ differing slightly in nuclear charge $z_{A}>Z_{B^{*}} \quad$ (These curves 
are actually appropriate for analyzing $\mathrm{K}$-shell excitations.) In region I, two o states run approximately parallel for some distance. In such a region a transition can take place even though there is no curve crossing. The coupling matrix element can be assumed to have an exponential dependence on separation, and the coupling between the two levels is of ten referred to as Demkov $(17,18)$ coupling. In region $I I$, a crossing occurs between the $2 \mathrm{p} \sigma$ and $2 \mathrm{p}_{\pi}$ levels. Similar crossings between levels of different angular momentum symmetry can occur at various internuclear separations, and the coupling between the relevant levels is collectively referred to as rotational coupling. (In region II, where the crossing takes place in the united atom limit, it is sometimes termed united atom coupling.) For some time it was thought that because of the different angular momentum symmetries involved, coupling of the two states would be unimportant at these crossings; however, because the internuclear line is rotating during the collision process, there is actually an angular momentum coupling between the levels. In fact, the $2 p_{\sigma}-2 p_{\pi}$ transition represents an extremely important process for $K$-shell excitation in heavy ion collisions. Finally, in region III we have a case where two $\sigma$ states cross. These are called radial couplings; the coupling is due to the radial motion, and the cross sections can be estimated by the we11-known Landau-Zener formula $(19,20)$. It should be noted that electron promotion to higher orbitals will take place only if an actual vacancy exists in these orbitals; otherwise, the Pauli principle excludes such transitions. Thus, for example, for atoms with $\mathrm{Z}>10$ in which the $\mathrm{L}$ shells are complete, the $2 \mathrm{p}_{\sigma}-2 \mathrm{p}_{\pi}$ rotational interaction cannot occur unless a previous collision has removed one or more L-shell elec'rons. 
Consider once more Figure 9, the Fano-Lichten diagram for $\mathrm{Ar}^{+}-\mathrm{Ar}$ levels and, in particular, the $4 f_{\sigma}$ molecular level as the internuclear separation is decreased slightly from the value $R_{s} \approx 0.5$, where it begins its sharp rise. During a comparatively small change in internuclear separation, the level makes crossings with a number of states to which transitions can occur through either radial or rotational coupling. Such a situation exists for many collision systems and, in fact, a particular level may pass through a mantfold of states within a small region of internuclear separations. Under these conditions the probability is close to $100 \%$ that some transition will occur and that at least one of the atoms will be in an excited or ionized state following the collision.

\section{ENERGY THRESHOLDS FOR INNER SHELL EXCITATION}

We consider now, in a very approximate manner, the energy thresholds required for inner-shell excitations. In general, whether we think in terms of the situation involving a large number of crossings in a restricted range of internuclear separations characteristic of L-shell interpenetration effects or of the characteristic $\mathrm{K}$-shell mechanism involving the $2 \mathrm{p}_{\sigma}-2 \mathrm{p}_{\pi}$ transition, one can say roughly that the cross section approaches a value that is of the order of $\pi_{S} R_{S}^{2}$, where $R_{S}$ is a critical internuclear separation (basically the sum of the relevant interpenetrating shell radii). To reach this distance of approach, the collision energy must be higin enough to overcome the repulsive force keeping the two nuclei apart and also to supply the energy I required for excitation (or ionization). One can roughly 
characterize the magnitude and energy dependence of the cross section by a crude lormula:

$$
\sigma \approx \pi R_{s}^{2}\left(1+\frac{I_{s}+Z_{1} Z_{2} e^{2 / R} s}{E}\right)=\pi_{S} R^{2}\left(1+\frac{E S}{E}\right),
$$

where $E$ is the collision energy, and $z_{1}$ and $z_{2}$ are the effective nuclear charges of the target and projectile atoms, respectively (nuclear charge reduced by the effective screening of the electron shells inside $R_{s}$ ).

For inner-shell interactions, assuming $z_{1} \approx z_{2}=z$, the repulsive potential $\mathrm{Z}_{1} \mathrm{Z}_{2} e^{2} / R_{\mathrm{s}}$ (which is proportional to $\mathrm{Z}^{3}$, since $\mathrm{R}_{5}$ is proportional to $\frac{1}{\mathrm{Z}}$ ) dominates $I_{s}$ (which is proportional to $z^{2}$ ). The threshold for the inner-shell excitation process occurs, therefore, at a collision energy many times larger than the characteristic inner-shell energy I and the cross section gradually approaches $\pi_{S}^{R}{ }_{S}^{2}$ as the energy increasis further.

For outer shell transitions, because of screening effects, the nuclear repulsion loses its importance, and the threshold is determined essentially by $I_{s}$, the outer shell excitation (or ionization) potential.

ENERGY DEPOSITION IN INNER-SHELL PROCESSES

We proceed now to obtain a rough estimate of the relative amount of energy expended in inner-shell excitation and ionization as momncred to outer-shell processes. For a given shell at energies well above threshold, the cross section for excitation of a given electron approaches the quantity $\sigma_{s}=\pi R_{s}^{2}$. The amount of energy that can be deposited in a given shell labeled by the principal quantum number $s$ is related to $\mathrm{N}_{\mathrm{s}}$, the number of 
electrons in the she11, and also to the relevant excitation (or ionization) potential. This we can approximate in the hydrogenic form as

$$
I_{s} \cong \frac{e^{2} z_{s}^{2}}{2 a_{0} s^{2}}
$$

The simplest approximation available for $R_{s}$ is

$$
\mathrm{R}_{\mathrm{s}} \cong 2 \mathrm{a}_{\mathrm{o}} \mathrm{s} / \mathrm{Z}_{\mathrm{s}}
$$

The energy per atom deposited in a given shell by ionization will then be given roughly by

$$
E_{s}=\sigma_{s} N_{s} I_{s} \cong \pi R_{s}^{2} I_{s} N_{s} \cong 2 \pi a_{0} e^{2} N_{s}
$$

This expression should be used on $1 \mathrm{y}$ for order-of-magnitude estimates and only at energies several times the effective threshold given by $E_{s}$ in Eq. (1). It is useful, however, in providing a measure of the amount of energy deposited in a given shell by relatively fast bombarding particles. Thus, when the collision energies get sufficiently large, the fraction of the energy absorbed in exciting the shell is determined by the number of inner-shell electrons compared to the number of outer-shell electrons. On the basis of this very rough model, one could say that, in a biological medium containing nitrogen, oxygen, or carbon, something of the order of 10 to $20 \%$ of the energy associated with primary ionization is 11 kely to go into K-shell excitation processes in these atoms and the rest into outer-shell excitations. This is quite a significant energy deposition. The vacancies produced will result in Auger transitions, with consequent ejection of large numbers of energetic electrons with a high potential for producing localized biological damage. 


\section{CONCLUDING REMARKS}

We have briefly outlined current understanding of shell interpenetration excitation and ionization phenomena and other excitation processes associated with heavy ion-atom collisions at relatively low energies. In a sense, one purpose of this paper has been to counter the common impression that, once the velocity of a charged particle falls below the velocity of atomic valence electrons in the medium, collisions are largely elastic and rarely lead to electronic excitation. We have examined experimental evidence to the effect that low energy excitation cross sections in such systems are many orders of magnitude larger than the cross sections predicted on the basis of the conventional coulomb-ionization model. The deeper understanding of inner-shell excitation processes and of electron capture reactions that is being achieved will certainly find application in several areas of radiation research. For example, on the basis of the results discussed here, it seems clear that the energy loss of a charged particle in matter should have much greater statistical fluctuations (straggling) than those predictable from earlier conceptions. More generally, findings such as these will aid immeasurably in elucidating the energy-loss mechanisms associated with the Bragg peak. Finally, the demonstrated importance of inner-shell excitation processes in these collisions focuses the spotlight on the attendant Auger processes with their high potential for localized radiation damage via coulombic explosions and the production of high-LET electrons. 
REFERENCES

1. U. Fano, Radiation Research 1966, Proc. I I International Congress Radiation Res., Cortina d'Ampezzo, 1966; G. Silini, Ed. (North Holland, Amsterdam, 1967), pp. 20-42, 68-95.

2. V. V. Afrosimov, Yu. S. Gordeev, M. N. Panov, and N. V. Fedorenko, Zh. Tekhn. Fiz. 34, 1613, 1624, 1637 (1964) [Sov. Phys.-Tech. Phys. $9,1248,1256,1265(1965)]$.

3. E. Everhart and Q. C. Kessel, Phys. Rev. Letters 14, 247 (1965).

4. Q. C. Kessel, A. Russek, and E. Everhart, Phys. Rev. Letters 14, 484 (1965).

5. U. Fano and W. Lichten, Phys, Rev, Letters 14, 627 (1965).

6. Q. C. Kessel and B. Fastrup, Case Studies in Atomic Physics 3, 139 (1973).

7. J. D. Garcia, R. J. Fortner, and T. M. Kavenaugh, Rev. Mod. Phys . 45, 111 (1974).

8. Proceedings of the International Conference on Inner Shell Ionization Phenomena and Future Applications (U.S. Atomic Energy Commission, Technical Information Center, Oak Ridge, Tenn., 1972).

9. w. Brandt, p. 948 in Ref. 8.

10. E. Merzbacher, p. 915 in Ref 8.

11. W. Brandt and R. Laubert, Phys. Rev. Letters 24, 1037 (1970).

12. G. N. Ogurtsov, Rev. Mod. Phys, 44, 1 (1972).

13. V. V. Afrosimov, Yu. S. Gordeev, A. M. Polyanskil, ant A. P. Shergin, Zh. Eksp. Teor, Fiz. 57, 806 (1969) [Sov, Phys, JETP 30, 441 (1s:?)].

14. B. Fastrup, G. Hermann, and K. J. Smith, Phys. Rev. A 3, 1591 (1971). 
15. Q. C. Kesse1, P. H. Rose, and L. Grodzins, Phys. Rev. Letters 22, 1031 (1969).

16. W. Lichten, Phys. Rev.. 164, 131 (1967).

17. Yu. N. Demkov, Zh. Eksp. Teor. Fiz. 45, 195 (1963) [Sov. Phys. JETP 18, $138(1964)]$.

18. R. E. Olson, Phys, Rev, A 6 , 1822 (1972).

19. L. D. Landau, Phys. Z. Sowjetunion 2, 46 (1932).

20. C. Zener, Proc. Roy Soc. (London), A137, 696 (1932). 


\section{Figure Captions}

Fig. 1. Reduced plot of $\mathrm{K}$-shell ionization cross sections vs. incident particle energy for structureless charged particles on various atoms. The theoretical curve (solid line) is derived from plane wave born approximation theory with corrections made for effects due to coulomb deflection of the projectile and variation of K-shell binding with internuclear distance. From W. Brandt, Ref. 9.

Fig. 2. Reduced plot of $\mathrm{K}$-shell ionization cross sections vs, incident particle energy for both structureless charged particles and heavy ions (with associated electron shell structure) on various atoms. The theoretical curve is the same as in Fig. 1. From W. Brandt, Ref. 9.

Fig. 3. Cross sections for K-shell excitation of atoms by incident heavy ions plotted in terms of the reduced parameters used in the binary encounter theory. E and $u$ are respectively the ion energy and the $K$ e:ectron binding energy, $\lambda$ is the ratio of the projectile to electron mass, and $z_{1}$ is the projectile atomic number. The solid curve is derived from binary encounter theory. From Garcia, et al., Ref. 7.

Fig. 4. Cross sections for L- and M-shell excitation of atoms by incident heavy ions plotted in terms of the reduced parameters used in the binary encounter theory. The description is the same as that for Fig. 3. From Garcia, et al., Ref. 7 . 
Fig. 5. Comparison of Ne K-she11 Auger electron spectra produced by (a) $1.5 \mathrm{keV}$ photons, (b) $3.2 \mathrm{keV}$ electrons, (c) $300 \mathrm{keV}$ protons, and (d) $200 \mathrm{keV}$ $\mathrm{Ne}^{+}$ions. From Ogurtsov, Ref. 12 .

Fig. 6. The relative cross sections for producing zero, one, or two $\left(Q_{I}, Q_{I I}\right.$, or $\mathrm{Q}_{\mathrm{III}}$ ) L-shell vacancies in $\mathrm{Ar}^{+}+\mathrm{Ar} \rightarrow \mathrm{Ar}^{2+}+\mathrm{Ar}^{2+}+3 e$ collisions plotted as a lunction of $Q$ for $25 \mathrm{keV}, \theta=16^{\circ}$ collisions. The quantities $Q$ and $\theta$ are the inelastic energy loss and scattering angle, respectively. From Afrosimov, Ref, 13.

Fig. 7. The relative probabilities $P_{i}$ for producing zero, one, or two (I, II, III) L-shell vacancies plotted as a function of distance of closest approach for $50 \mathrm{keV} \mathrm{Ar}{ }^{+}$- Ar collisions. From Fastrup, et al., Ref. 14.

Fig. 8. The average inelastic energy loss $\bar{Q}$ for $\mathrm{Ar}^{+}$- Ar collisions plotted as a function of distance of closest approach with the incident energy as a parameter. From Kessel, et al., Ref. 15.

Fig. 9. Semi-quantitative diabatic molecular orbital energy level diagram for the $\mathrm{Ar}_{2}$ molecule as proposed by Fano and Lichten. Fram Fano and Lichten, Ref. 5 .

Fig. 10. Schematic representation of the lowest one-electron energy levels of two atoms $A$ and $B$ differing slightly in nuclear charge with $z_{A}>z_{B}$. 


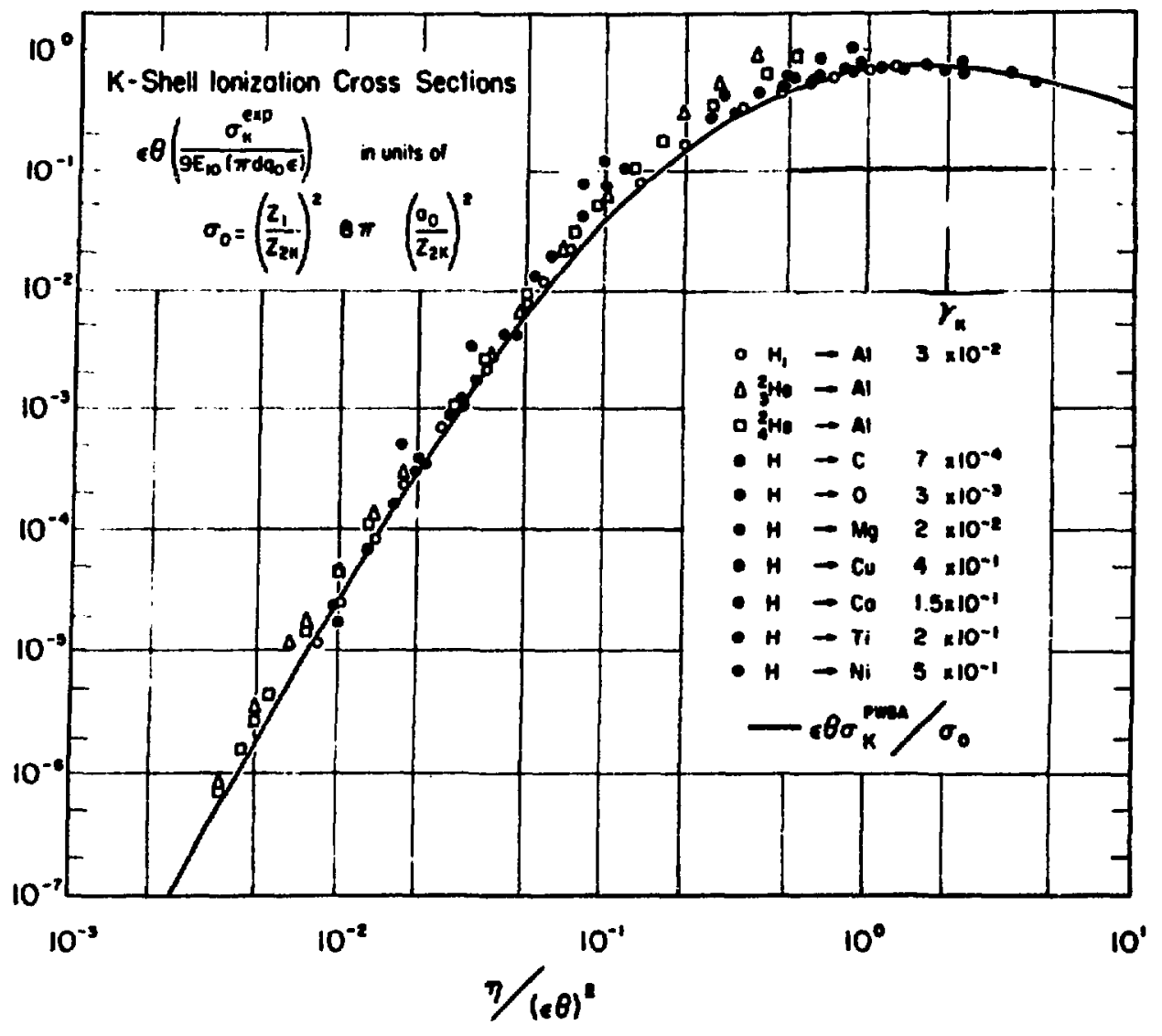

Figure 1 


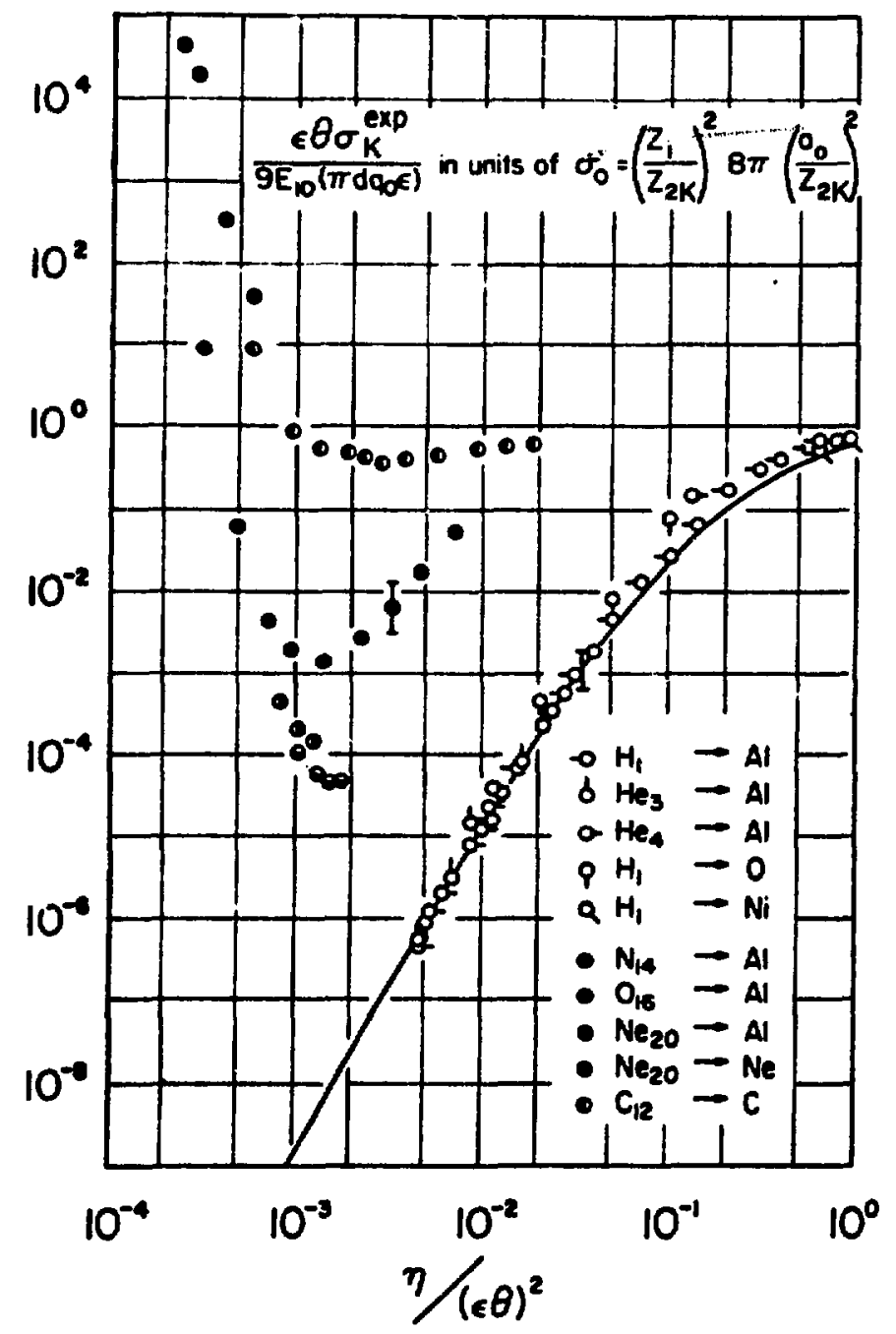

Figure 2 


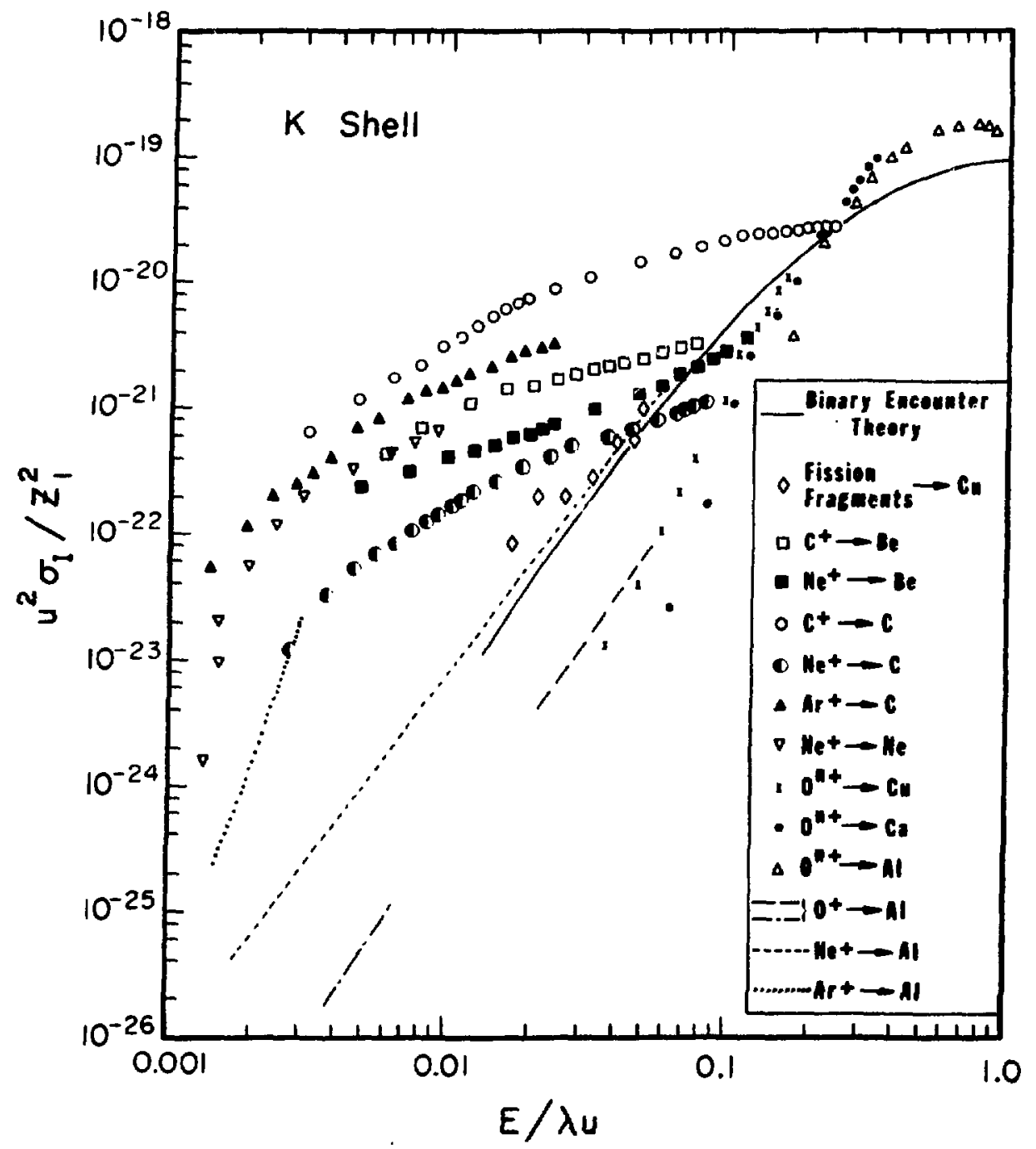

Figure 3 


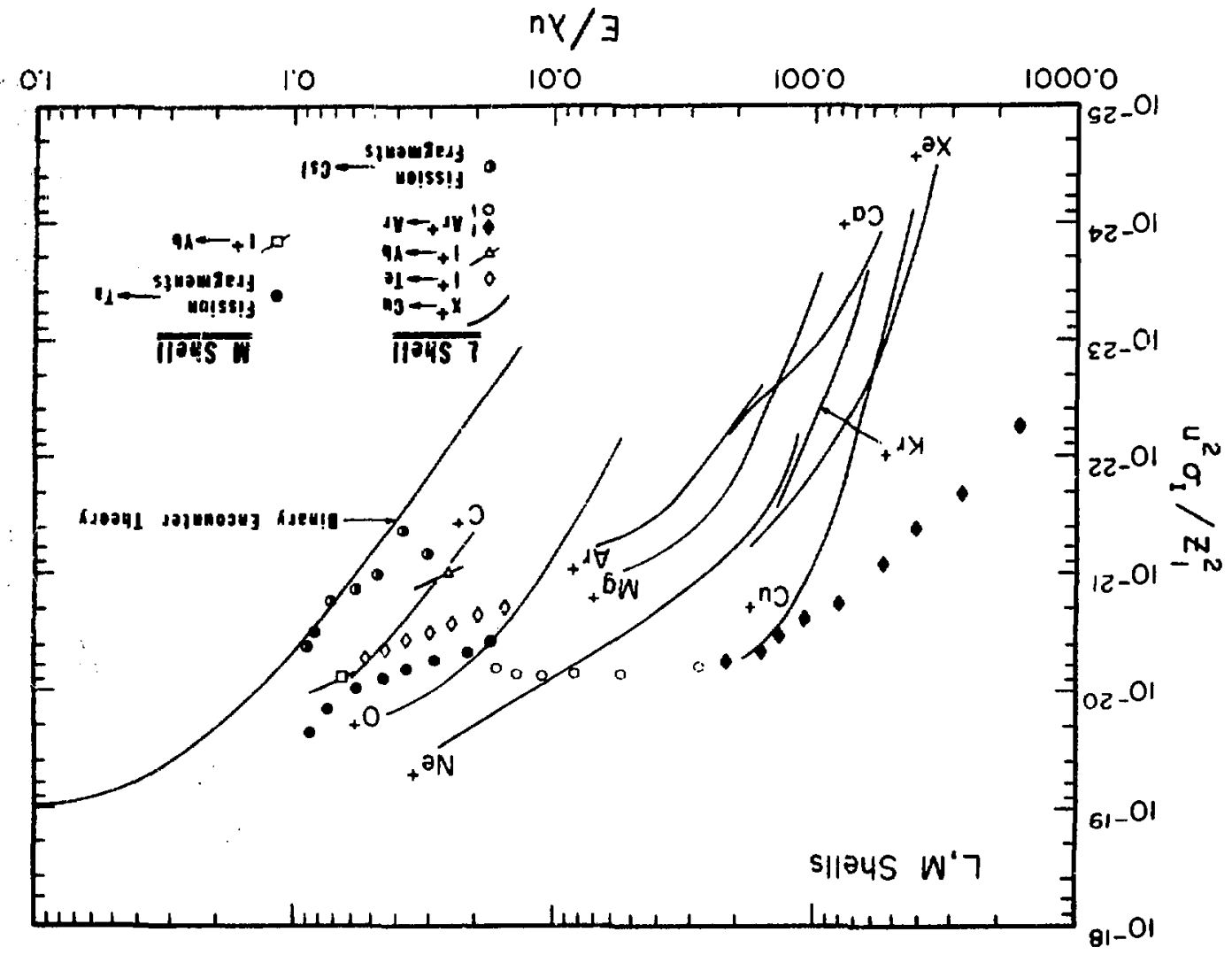




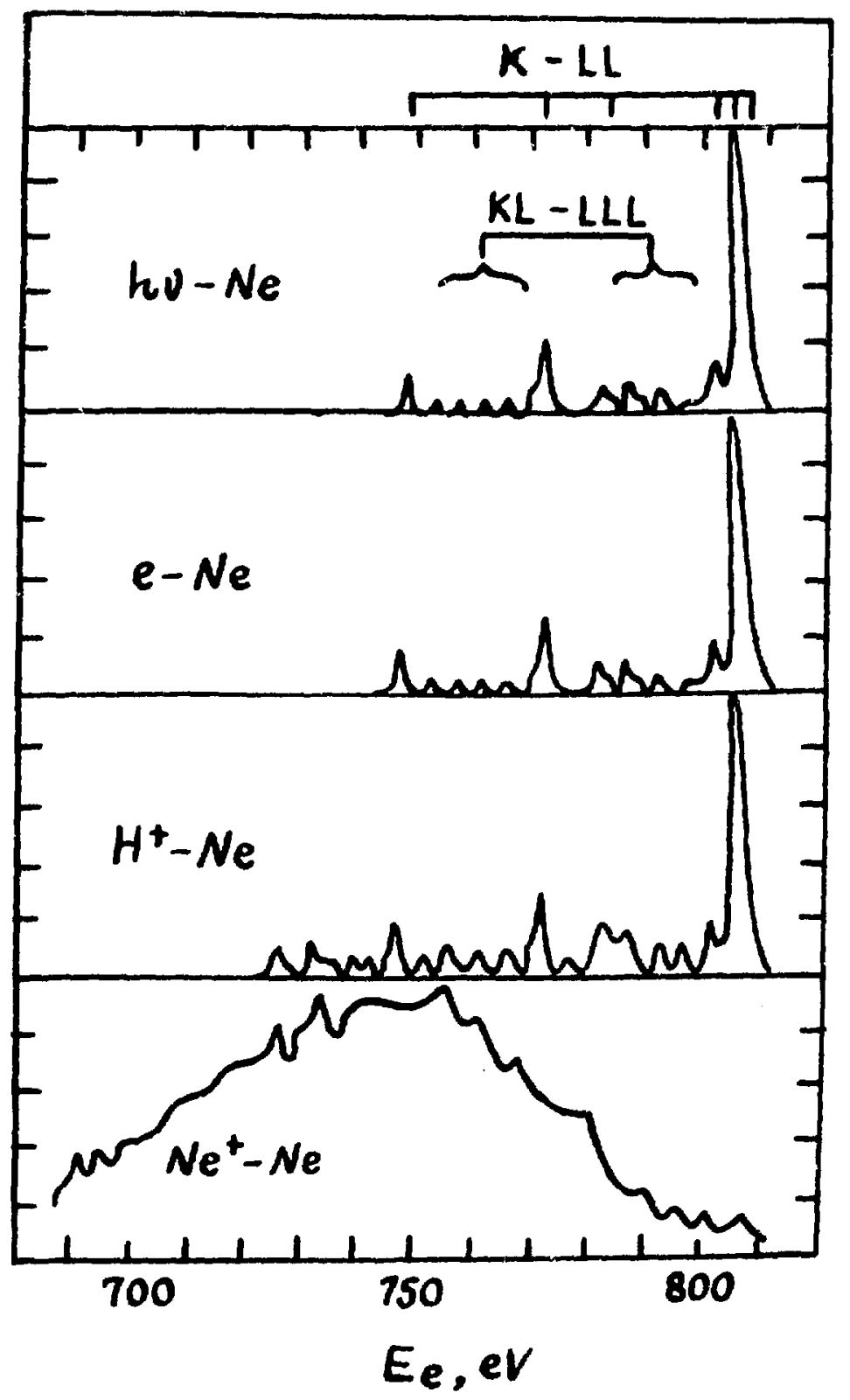

Figure 5 


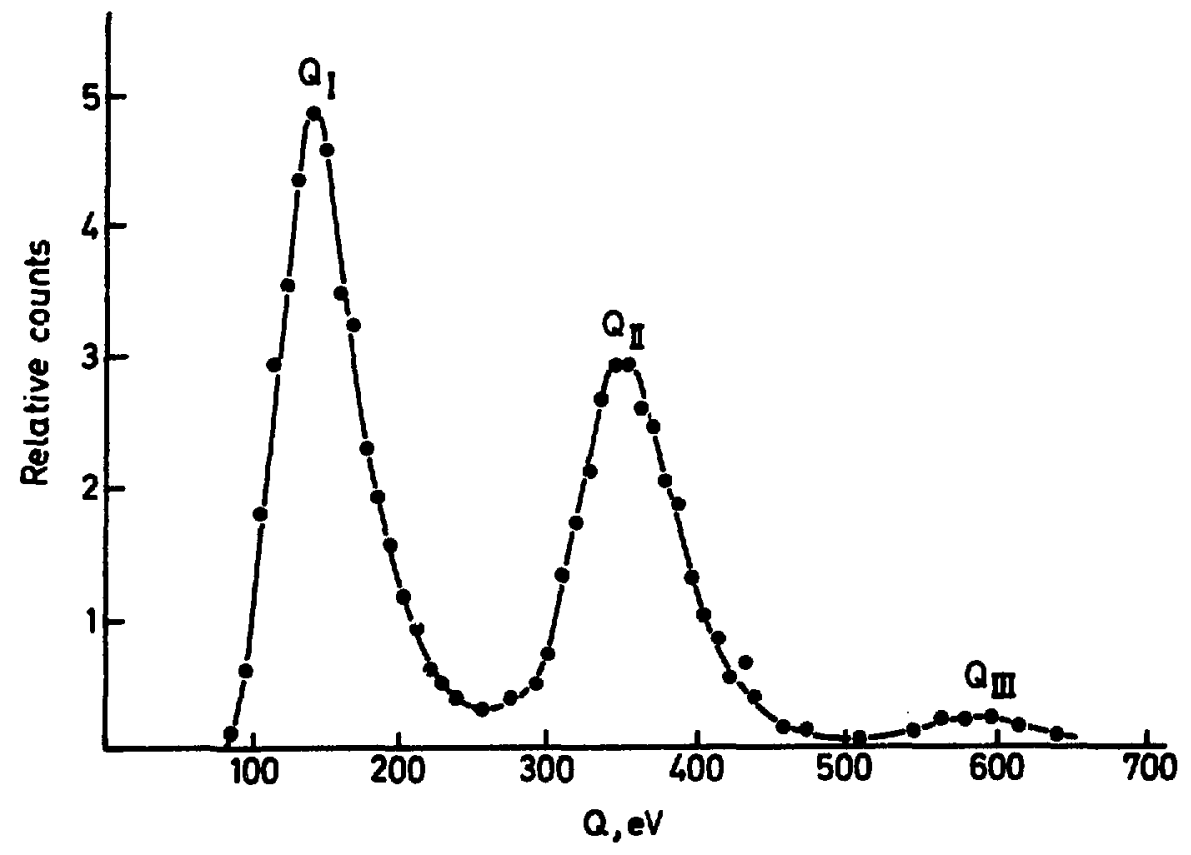

Figure 6 


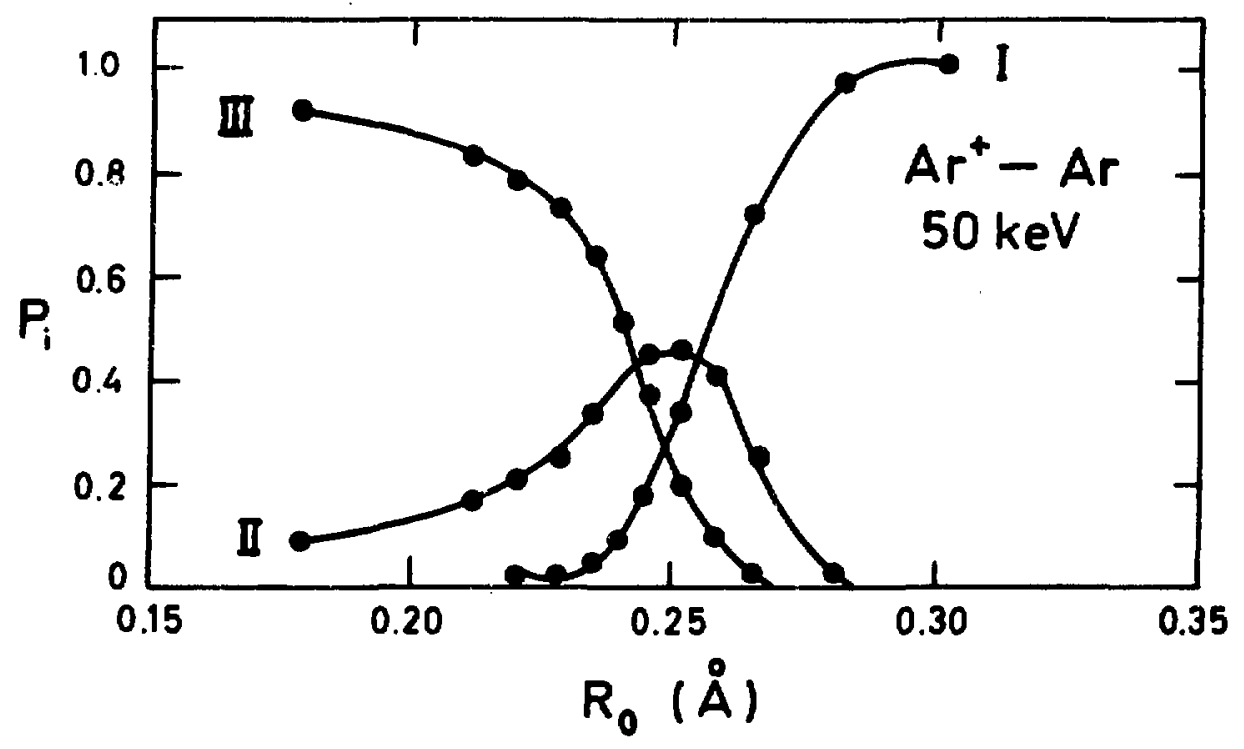

Figure 7 

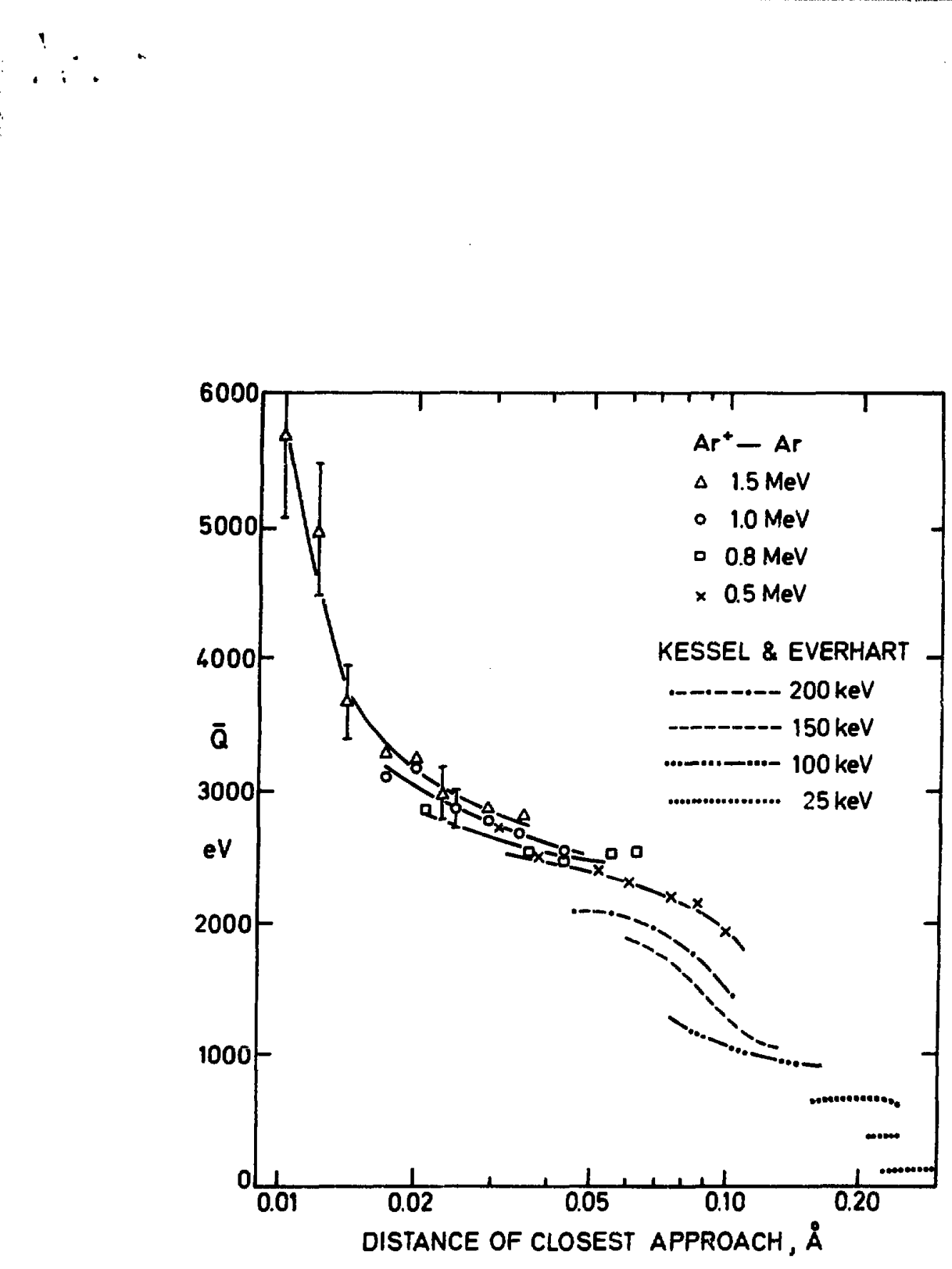

Figure 8 


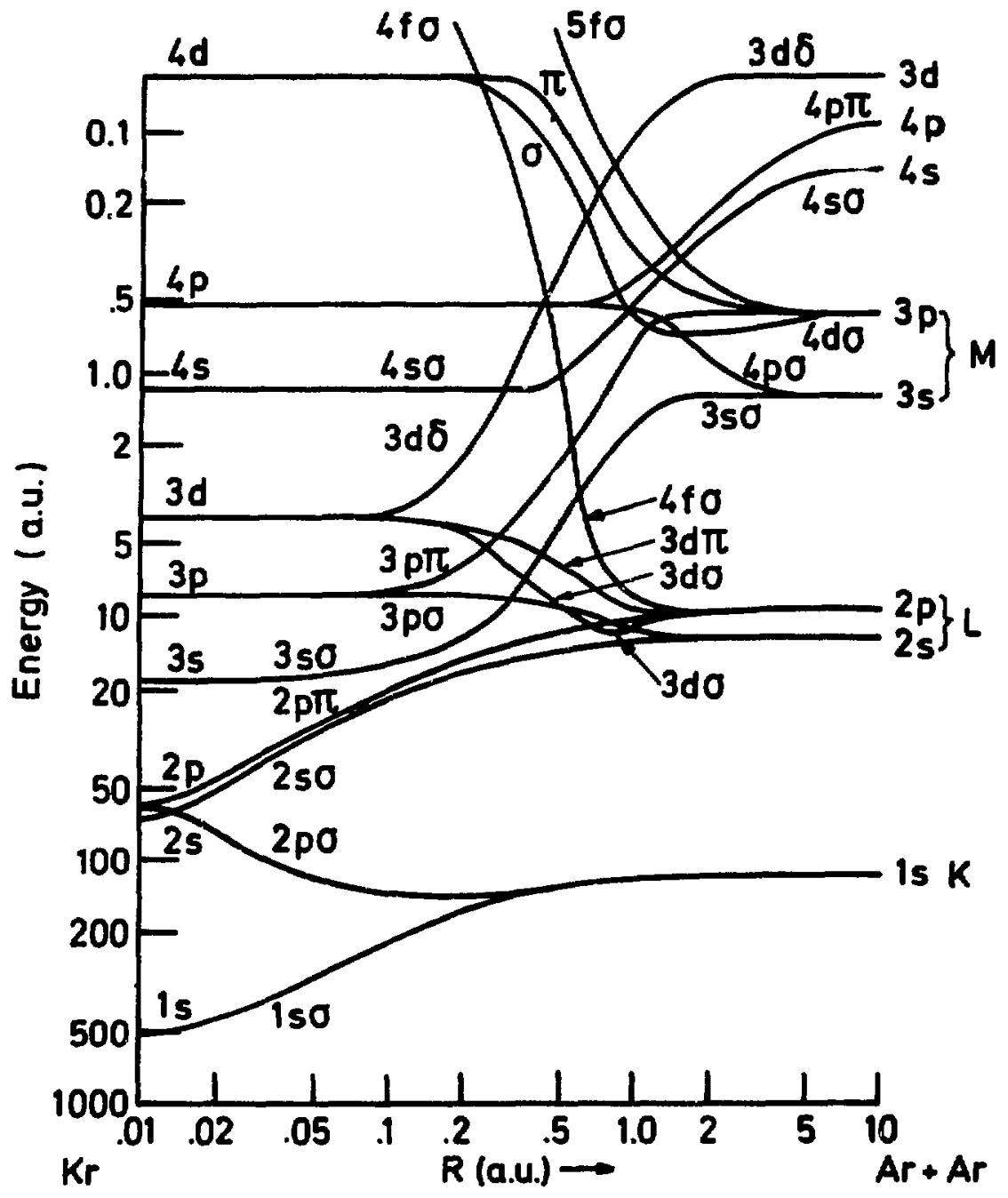

Figure 9 
